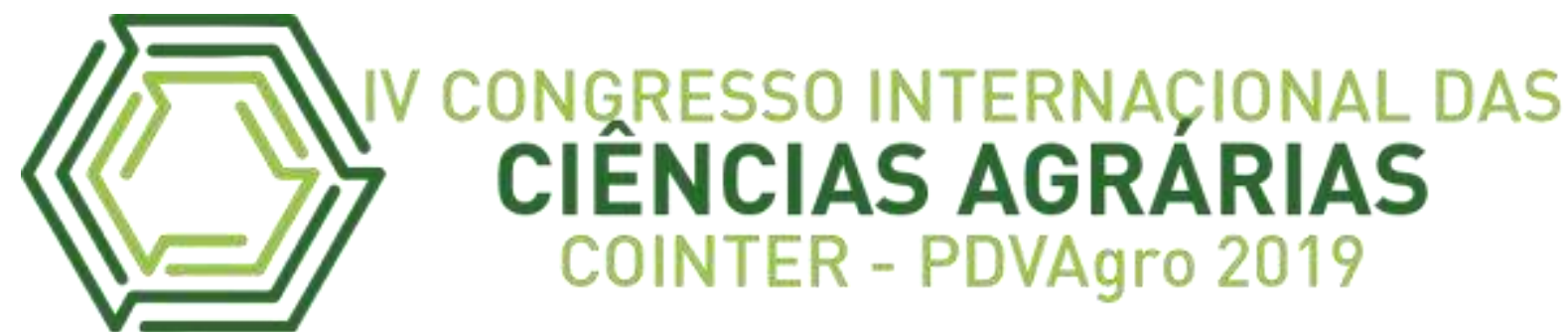

\title{
MONITORAMENTO DA PROSPECÇÃO CIENTÍFICA E TECNOLÓGICA DA VINAGREIRA
}

\author{
Apresentação: Comunicação Oral
}

Katiury Castro Cavalcante ${ }^{1}$; Álvaro Itaúna Schalcher Pereira ${ }^{2}$; Gladson Diniz Pinheiro ${ }^{3}$;
Oswaldo Palma Lopes Sobrinho ${ }^{4}$; Francisco Adelton Alves Ribeiro

DOI: https://doi.org/10.31692/2526-7701.IVCOINTERPDVAgro.2019.0004

\begin{abstract}
Resumo
A espécie Hibiscus sabdariffa L. (hibisco, quiabeiro-azedo, rosélia, vinagreira) é uma planta herbácea e arbustiva de poderoso potencial medicinal com origem na Índia e trazida ao Brasil por meio do tráfico de escravos. A presente pesquisa tem por objetivo descrever utilizando técnicas de prospecção científica e tecnológica informações sobre a hortaliça da espécie Hibiscus sabdariffa L. presentes nos bancos de dados nacionais e internacionais avaliando seus aspectos químicos e aplicações farmacológicas/medicinais. Para isso, foram realizadas buscas nas bases de dado científicas Web of Science e Scopus e nos bancos de patentes: Instituto Nacional da Propriedade Industrial (INPI) e World Intellectual Property Organization (WIPO). Das bases estudadas, a Scopus e WIPO foram as que obtiveram mais resultados em número de publicações e patentes depositadas. A Nigéria foi o país que mais publicou sobre o hibisco no período de 2007-2017, seguida da Índia e Taiwan. A China foi o país com maior número de patentes registradas, detendo $53 \%$ dos resultados. O Brasil apresentou uma baixa proporção no número de depósitos. Os documentos encontrados na WIPO se enquadraram nas seções $\mathrm{A}$ e $\mathrm{C}$ da classificação CIP, com a maioria deles inclusos na subclasse $\mathrm{A} 61 \mathrm{~K}$ (preparações para finalidades médicas, odontológicas ou higiênicas). Considerando o fato de que quanto mais técnicas são descobertas, maior é a possibilidade da construção de novos saberes, esta pesquisa mostrou-se relevante e inovadora, pois através dos estudos de prospectivos, o conhecimento coletado sobre a espécie Hibiscus sabdariffa L. pode servir de base para pesquisadores que pretendem desenvolver trabalhos de inovação ou apenas obterem informações das características, composição química e propriedades farmacológicas da planta, além de proporcionar um panorama acerca dos avanços científicos dessa linha de pesquisa.
\end{abstract}

Palavras-chave: compostos químicos, patente, Hibiscus sabdariffa L.

\footnotetext{
Abstract

1 Licenciada em Química, Instituto Federal do Maranhão - Campus Codó (IFMA). E-mail: katiurycavalcante@hotmail.com

2 Professor Doutor, Orientador, Instituto Federal do Maranhão - Campus Codó (IFMA). E-mail: alvaro.pereira@ifma.edu.br

${ }^{3}$ Licenciado em Química, Instituto Federal do Maranhão - Campus Codó (IFMA).

${ }^{4}$ Engenheiro Agrônomo pelo IFMA/Campus Codó, Mestrando em Ciências Agrárias - Agronomia, Instituto Federal Goiano - Campus Rio Verde (IF Goiano). E-mail: oswaldo-palma@ hotmail.com

5 Professor Doutor, Coorientador, Instituto Federal do Maranhão - Campus Codó (IFMA). E-mail: adelton@ifma.edu.br
} 
This research aims to describe using techniques of scientific and technological forecasting information about the vegetable species Hibiscus sabdariffa L. present on the banks of national and international data evaluating its chemical aspects and pharmacological/medical applications. For this, searches were conducted in scientific data bases Web of Science and Scopus and patent banks: National Institute of Industrial Property (INPI) and World Intellectual Property Organization (WIPO). The bases studied, Scopus and WIPO were obtained which results in more number of publications and patents filed. Nigeria was the country that published on hibiscus in the 2007-2017 period, followed by India and Taiwan. China was the country with the highest number of registered patents, holding $53 \%$ of the results. Brazil has a low proportion in the number of deposits. Documents found in the WIPO classified into sections $\mathrm{A}$ and $\mathrm{C}$ of the IPC classification, with most of them included in subclass A61K (preparations for medical, dental or hygienic purposes). Considering the fact that the more techniques are discovered, the greater the possibility of building new knowledge, this research proved relevant and innovative, because through prospective studies, the knowledge collected about the species Hibiscus sabdariffa L. can serve as This is the basis for researchers who want to develop innovative work or just get information on the characteristics, chemical composition and pharmacological properties of the plant, and provide an overview of the scientific advances of this line of research.

Keywords: chemical compounds, patent, Hibiscus sabdariffa L.

\section{Introdução}

O Brasil é o país detentor da flora mais abundante do globo terrestre com cerca de $15 \%$ a $20 \%$ do total de espécies registradas. Além disso, o território brasileiro se destaca também, pela presença de variados biomas (Amazônia, Mata Atlântica, Cerrado) com quantidades significativas de espécies vegetais endêmicas (CORADIN; SIMINSKI; REIS, 2011).

Os estudos prospectivos a este constituem uma poderosa ferramenta na coleta, análise, interpretação e agregação de valor às informações do presente, sendo possível preservar o conhecimento coletivo e elaborar estratégias para alcançar o progresso na inovação tecnológica (GALVÃO, 2009). Acrescenta-se que essa linha de pesquisa aqui desenvolvida, vem a auxiliar na identificação de possíveis oportunidades e necessidades do futuro no meio técnico e social culminado em avanços tecnológicos e científicos no uso da vinagreira (Hibiscus sabdariffa).

A pesquisa fundamenta-se no uso da prospecção científica e tecnológica para o mapeamento de informações desenvolvidas em torno da espécie Hibiscus sabdariffa L. analisando suas potencialidades farmacológicas e evolução de competências tecnológicas em bases de dados nacionais e internacionais. Pretende-se colaborar com as pesquisas de inovação por meio de informações coletadas sobre os principais estudos da vinagreira na área da Química e Farmacologia no período equivalente a 2007-2017. 


\section{Metodologia}

A prospecção científica de aspecto quantitativo foi desenvolvida, exclusivamente, a partir de artigos publicados nas bases de dados presentes no Portal de Periódicos da CAPES (Coordenação de Aperfeiçoamento de Pessoal de Nível Superior), e para a revisão qualitativa utilizou-se, além dessas publicações, trabalhos de outras espécies como monografias, dissertações de mestrado e doutorado, livros e manuais.

Foram selecionados artigos na Scopus e Web of Science - Coleção Principal (Clarivate Analytics), utilizando como descritores o gênero (Hibiscus), a espécie (Hibiscus sabdariffa L.) e o nome vulgar em território nacional (vinagreira). Os termos acima citados foram pesquisados na opção "title, abstract and keywords" que em português faz referência aos campos de pesquisa que incluem título, resumo e palavras-chave, respectivamente. Durante a coleta de dados foi estabelecido um prazo que corresponde aos dez últimos anos de publicações científicas (2007-2017). Outros anos que chamaram atenção quanto ao número e relevância das pesquisas foram citados no decorrer do trabalho. Quanto ao tipo de documento, priorizou-se apenas artigos e resumos nos idiomas Português, Inglês e Espanhol. Assim, foram selecionados todos os periódicos publicados em revistas de território nacional e internacional.

Para atingir os objetivos desta pesquisa o monitoramento científico foi delimitado aos seguintes eixos: 1 - Farmacologia, Toxicologia e Farmácia (Pharmacology, Toxicology and Pharmacy) e 2 - Registro de patentes nas bases nacionais e internacionais. Entretanto, alguns periódicos aplicados em outras áreas de conhecimentos foram mencionados nos resultados e discussões para complementar o trabalho. Após os dados já filtrados, foram analisados somente os artigos e resumos da base de dado com o maior número de publicações sobre a espécie Hibiscus sabdariffa, onde os mesmos foram organizados e armazenados para, posteriormente, serem correlacionados e classificados em software específico (Microsoft Excelß 2016). Em seguida, os gráficos e tabelas gerados permitiram a caracterização das patentes quanto: a) evolução anual de tecnologias desenvolvidas nos últimos dez anos; b) principais instituições/empresas detentoras; c) países depositantes.

Para finalizar a análise das patentes registradas, as mesmas foram caracterizadas de acordo com critérios adotados pelas CIP (Classificação Internacional de Patentes). Nesse 
momento, explanou-se as seções, classes e grupos, assim como subclasses e subgrupos os quais esses documentos se enquadravam. Descreveu-se também as invenções tecnológicas mais recentes sobre a espécie Hibiscus sabdariffa.

\section{Resultados e Discussões}

Os artigos e resumos que seguiram os critérios da busca foram totalizados e agrupados segundo a palavra-chave e a base de dado pesquisada como mostra na Tabela 1. Nota-se uma diferença no número de registros em ambas as bases científicas tanto de documentos relacionados ao gênero Hibiscus quanto de documentos direcionados a estudos químicos/farmacológicos da espécie Hibiscus sabdariffa. Na Web of Science foram encontrados 630 periódicos, sendo que aproximadamente 35,9\% desse total são de trabalhos referentes à espécie Hibiscus sabdariffa $\mathrm{L}$.

Tabela 1: Total de publicações em bases de dados científicas no período de 2007-2017.

\begin{tabular}{c|cc}
\hline Palavras - chave & Web of Science & Scopus \\
\hline Hibiscus & 402 & 1.454 \\
Hibiscus sabdariffa & 226 & 610 \\
Vinagreira & $2^{*}$ & $3^{* *}$ \\
Total & 630 & 2.067 \\
\hline
\end{tabular}

Legenda: * Artigos publicados apenas no ano de 2017.

** Artigos publicados entre os anos de 2016-2017. Fonte: Própria.

Para análise de informações da Tabela 1 foram selecionados apenas artigos/resumos encontrados na base de dados Scopus que estivessem relacionados ao nome científico da planta em questão. Assim, de 2.067 periódicos publicados nessa fonte, cerca de 610 foram analisados em gráficos quanto à área de conhecimento, coleções científicas, autores, países e anos. Desse total de documentos, $546(89,5 \%)$ são do tipo artigo e $64(10,5 \%)$ correspondem a resumos.

A fim de descrever os aspectos químicos e propriedades farmacológicas/medicinais da espécie Hibiscus sabdariffa foram filtrados apenas 83 trabalhos para revisão de literatura. Nesse sentido, na Figura 1, a primeira análise gráfica contemplou as principais áreas de conhecimento das publicações, que foram: Medicina (267), Farmacologia, Toxicologia e Farmácia (211), Bioquímica, Genética e Biologia Molecular (178), Agricultura e Ciências Biológicas (175) e Química (157). Observa-se o valor percentual de cada uma dessas áreas, com destaque para Medicina que representa $27 \%$ do total de publicações.

Figura 1: Número de publicações da espécie H. sabdariffa por área de conhecimento. 


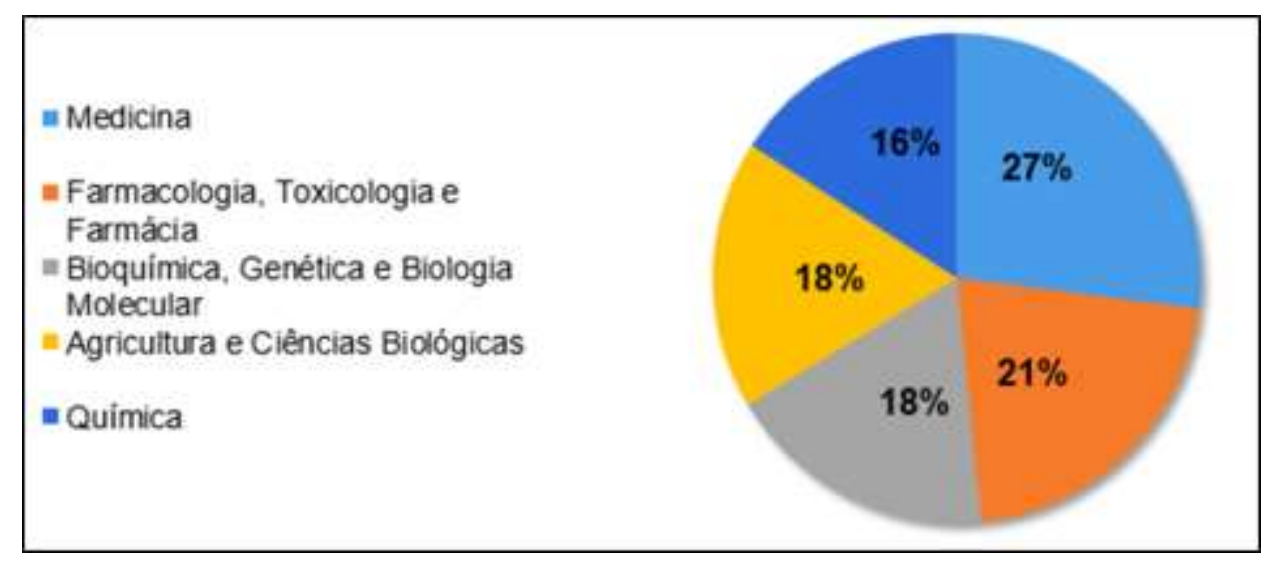

Fonte: Própria (2019)

Durante o período pré-estabelecido para a coleta de dados foram contabilizadas 159 coleções científicas que publicaram sobre a espécie Hibiscus Sabdariffa na base Scopus. Na Figura 2, estão representadas algumas dessas coleções, destacando a Journal of Ethnopharmacology (29), Food Chemistry (25), Journal Of Agricultural and Food Chemistry (14), Pakistan Journal Of Nutrition (13) e Journal Of The Science Of Food And Agriculture (12). Dos 29 artigos publicados na Journal Of Ethnopharmacology, todos estão enquadrados no eixo da Farmacologia, Toxicologia e Farmácia. Trata-se de uma revista com fator de impacto 2,981 e abrange trabalhos interdisciplinares com abordagem etnofarmacológica, etnobotânica ou bioquímica para o estudo das drogas indígenas. Assim, os artigos de revisão que contemplam as diversas atividades farmacológicas e toxicológicas de plantas também são inclusos nessa coleção.

Figura 2: Número de publicações da espécie $H$. sabdariffa por coleção científica.

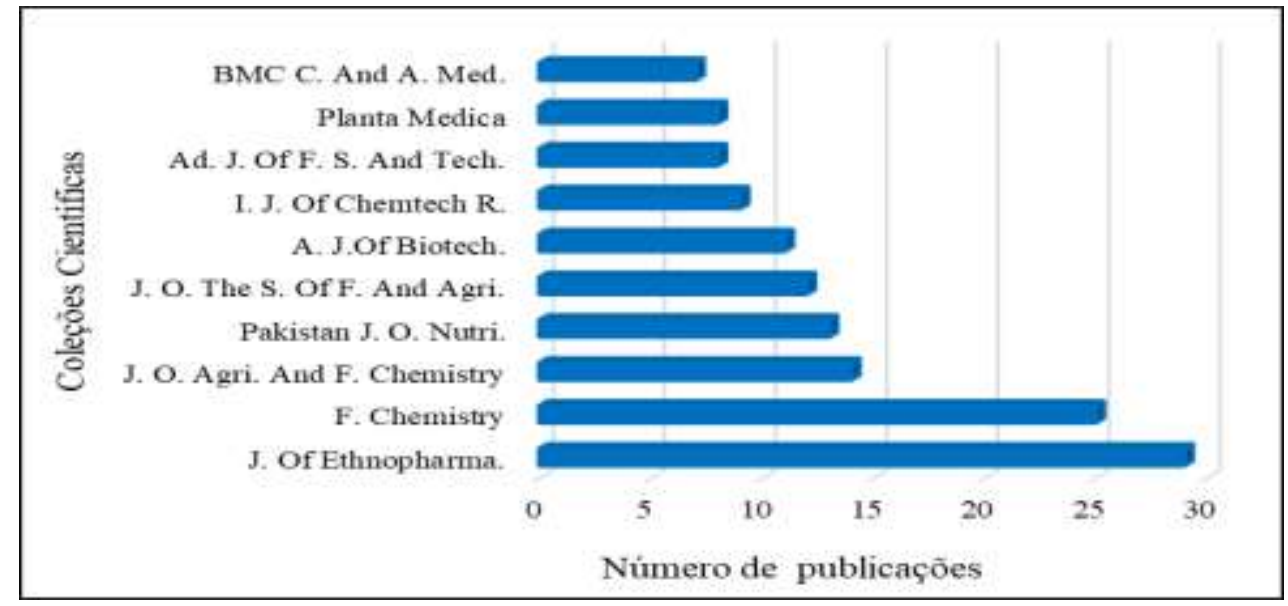

Fonte: Própria (2019)

A segunda colocada no ranking (Food Chemistry), com número de publicações foi igualmente expressivo à coleção anterior, apresenta fator de impacto de 4,529 e trabalha com “documentos de pesquisa originais sobre o avanço da química e bioquímica dos alimentos ou 
os métodos /abordagens analíticos usados" (Elsevier, 2018). Portanto, entre os critérios adotados por essa revista, os que mais se encaixam na linha da pesquisa sobre a espécie Hibiscus sabdariffa são: a) presença de componentes bioativos como antioxidantes; b) propriedades químicas, nutricionais, microbiológicas, fisiológicas e sensoriais do alimento (Elsevier, 2018).

As demais revistas em destaque, Journal Of Agricultural and Food Chemistry, Pakistan Journal Of Nutrition e Journal Of The Science Of Food And Agriculture apresentam fator de impacto 3,154 (ACS Publications, 2018); 0,29 (Asian Network for Scientific Information, 2018) e 2,463 (Wiley Online Library, 2018) respectivamente. Seguindo as análises foi obtido o resultado de 156 autores que publicaram sobre a espécie Hibiscus sabdariffa nas mais diversas revistas. A Figura 3, expressa o ranking dos principais escritores e a quantidade de suas contribuições para o desenvolvimento de pesquisas científicas. Os três primeiros autores destacaram-se pelo número de publicações.

Figura 3: Número de publicações da espécie H. sabdariffa por autores.

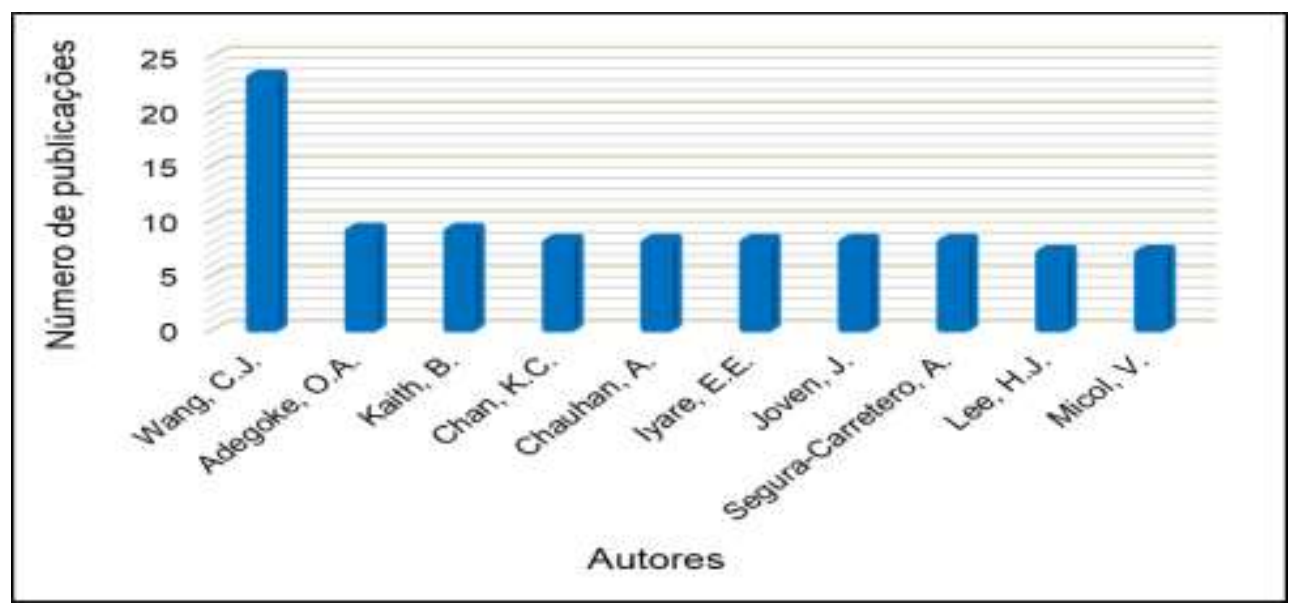

Fonte: Própria (2019)

A primeira colocação é ocupada pelo cientista e publicador Wang Chau Jong com 23 artigos produzidos. Em seguida, com uma quantidade bem menos expressiva de 9 artigos, encontraram-se empatados os autores Olajire A. Adegoke e Kaith, B. Quando delimitada apenas a esses 41 artigos, a pesquisa na base de dados Scopus determinou que cerca de $44 \%$ desse total são de documentos aplicados na área da Agricultura e Ciências Biológicas e 26\% na área da Química. Das contribuições de Wang Chau Jong e seus colaboradores constatou-se que 11 artigos foram publicados abordando aspectos químicos, bioquímicos e farmacológicos da espécie Hibiscus sabdariffa. O autor é de Taiwan e desenvolve trabalhos científicos desde 1986 a 2017. 
A distribuição de publicações a nível territorial é observada na Figura 4. A Nigéria (93) e a Índia (84) assumem o post de países com maior número de artigos publicados nos anos de 2007 a 2017. Na sequência encontram-se Taiwan (36), Egito (35), México (35), Malásia (31), Estados Unidos (28), Indonésia (26), Espanha (24), China (23), Tailândia (23), França (20) e em $13^{\circ}$ lugar o Brasil com 19 artigos. Os demais países que publicaram não foram registrados na Figura 4 dados a baixa proporção de resultados.

Figura 4: Número de publicações da espécie $H$. sabdariffa por país.

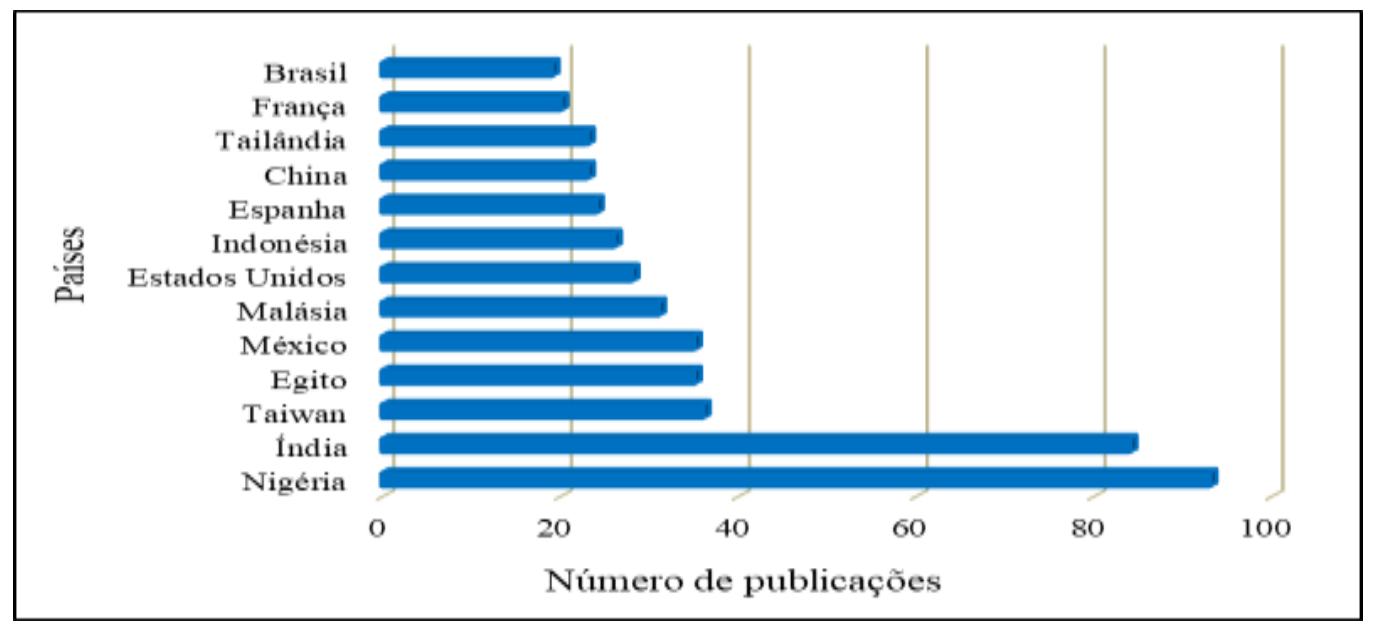

Fonte: Própria (2019)

O cultivo e o desenvolvimento de pesquisas sobre o hibisco na Nigéria e na Índia são destinados a vários fins. Dos artigos encontrados na base Scopus observou-se uma larga aplicação da espécie Hibiscus sabdariffa no desenvolvimento de medicamentos tradicionais para o tratamento de doenças renais, cardiovasculares e musculares, haja vista a baixa e média renda desses países. Assim, cerca de 38,9\% (68) dos artigos publicados em território nigeriano e indiano estão enquadrados na área classificada como "remédios". Os artigos publicados no Brasil ganham destaque na área da Química, Bioquímica e Biologia Molecular com 58\% (11) do total de registros. Os estudos apontam o uso do hibisco na medicina popular devido a suas atividades anti-inflamatória e antioxidante. Além disso, a planta também aparece aplicada no processo de perda de peso e proteção contra o dano hepático.

Mediante análise dos dados coletados, percebe-se uma evolução anual no número de publicações do gênero Hibiscus e da espécie Hibiscus sabdariffa no período de 2007 a 2017 (Figura 5). Levando em consideração apenas os 610 artigos publicados sobre a espécie, é possível observar um aumento significativo no desenvolvimento de trabalhos científicos entre os anos de 2011 e 2017.

Figura 5: Número de publicações do gênero Hibiscus e da espécie H. sabdariffa nos anos de 2007-2017. 


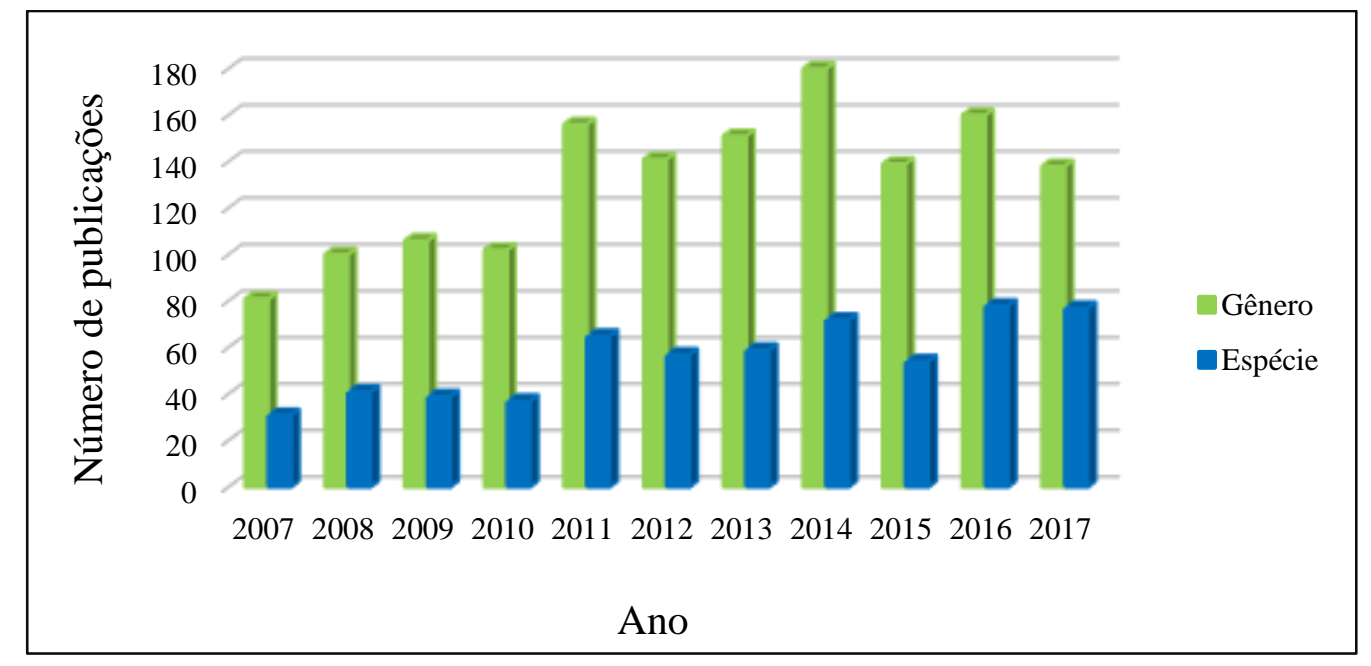

Fonte: Própria (2019)

De acordo com a Figura 5 o ano com mais publicações do gênero Hibiscus corresponde a 2014 com 11\% dos artigos. Já com relação à espécie do hibisco, o ano de destaque foi 2016 com 12,8\% do total de trabalhos. Os estudos encontrados na revisão de literatura científica ratificaram a utilização do hibisco na medicina popular em forma de chás, vinhos, vinagres, dentre outras bebidas nutritivas. O cálice, como já esperado é a parte vegetal mais procurada para elaboração de produtos com variadas aplicações fitoterápicas. Em seguida, o óleo extraído das sementes é largamente aplicado na prevenção e no controle de doenças hipertensivas ocasionadas pelo aumento dos níveis de LDL (Low Density Lipoprotein) no organismo. No Quadro 1 estão listados os principais usos medicinais do hibisco relatados em trabalhos científicos.

Quadro 1. Principais atividades farmacológicas da espécie H. sabdariffa L.

\begin{tabular}{|c|c|}
\hline \multicolumn{1}{|c|}{ Propriedades Farmacológicas } & Referências \\
\hline Antioxidante & Ali et al. (2003) \\
\hline Antimalárico & Ali et al. (1991) \\
\hline \hline Antiaterosclerótica & Liu et al. (2006) \\
\hline \hline Antiespasmódico & Ali et al. (1991); Sarr et al. (2009) \\
\hline \hline Antibacteriana/Antifúngica/Antiparasitária & Liu et al. (2006); Fullerton et al. (2011) \\
\hline \hline Anti-inflamatória & Kao et al. (2016) \\
\hline \hline Hepatoprotetora & Liu et al. (2006) \\
\hline \hline Hipocolesterolemiante & Lin et al. (2006); Gurrola-Diaz et al. (2010) \\
\hline \hline Uricosúrica & Al-Mamun et al. (2016) \\
\hline \hline Citotóxica & Gurrola-Diaz et al. (2010) \\
\hline \hline Antiobesidade & Alarcon-Alonso et al. (2012); \\
\hline Efeito diurético & Ajay et al. (2007) \\
\hline \hline Anti-hipertensiva & Liu et al. (2006) \\
\hline Efeito quimiopreventivo para o câncer & \\
\hline
\end{tabular}


A propriedade antioxidante atribuída ao cálice do H. sabdariffa foi citada também em uma série de estudos in vitro, além daqueles referenciados no Quadro 2 (HIRUNPANICH et al., 2005; OLALYE; ROCK, 2007; MOHD-ESA et al., 2010). Em pesquisas mais recentes, os efeitos antioxidantes e anti-inflamatórios do hibisco foram observados em experimentos com extratos metanólicos das folhas, nos quais a presença de compostos bioativos como o ácido caféico e ácido neoclorogênico contribuíram para o desempenho na redução do estresse oxidativo em modelos celulares e na enzima mieloperoxidase (MPO), principais agentes responsáveis na inflamação (KAPEPULA et al., 2017).

A atividade antibacteriana do cálice da planta mostrou-se eficiente no combate a várias cepas bacterianas in vitro, tais como Bacillus stearothermophilus, Micrococcus luteus, Clostridium sporogenes, Escherichia coli e Bacillus cereus (OLALEYE, 2007). O efeito antiEscherichia coli foi confirmado nos experimentos de Paim et al. (2017) que aplicando diferentes concentrações $(5,10,20$ e 30\%) do extrato hidroetanólico de H. sabdariffa em porções de carne moída contaminadas com E. coli. obteve redução logarítmica em praticamente todos os tratamentos, sendo o maior resultado observado na carne contendo $30 \%$ do extrato vegetal.

Conforme o Quadro 1, o efeito antiespasmódico do hibisco vem sendo analisado desde a década de 1990, com os estudos de Ali et al. (1991) que comprovaram a atuação do mesmo no controle das contrações musculares do útero, cadeia traquial e diafragma do rato, os quais retraem ritmicamente. A mesma propriedade foi posteriormente constatada por Sarr et al (2009) na indução do efeito relaxante de músculos localizados na região torácica de ratos, ocasionada principalmente pela ação biológica de polifenóis presentes na hortaliça.

O extrato do cálice do hibisco é também empregado na redução de danos hepáticos e renais induzidos in vitro por toxinas como a tiociacetamida (TAA), azitioprina, tetracloreto de carbono, cádmio e irradiação (LIU et al., 2010). Em exemplo disso, o experimento realizado com ratos de laboratórios por Ezzat et al (2016) demonstrou que a variedade de antocianinas presentes nos cálices do $H$. sabdariffa apresentaram funções hepatoprotetoras incluindo a atividade de necrose tumoral e restauração da estrutura hepática em até $70 \%$. Em outro caso, a aplicação do extrato do cálice com diferentes concentrações de antocianinas (50, 100 e 200 $\mathrm{mg} / \mathrm{kg})$, apresentou a mesma eficácia que o uso do lisonopril (10 mg/kg) em ratos com doenças renais provocadas por altas dosagens de adenina (ALI et al., 2017).

Recentemente, os polifenóis de extratos foliares do hibisco também demonstraram 
atividade anticancerígena na inibição de tumores de próstata em ratos (Lin et al., 2012) e atividade anti-hipertensiva no controle da pressão arterial e aumento do diâmetro dos vasos sanguíneos (AJAY et al., 2007; INUWA et al., 2012). O resultado para a busca de patentes nos dois bancos de dados analisados, sem prazo estabelecido, foi organizado e agrupado como mostra na Tabela 2. Foram encontradas 8 patentes na base de dado nacional INPI (Instituto Nacional da Propriedade Industrial), sendo 3 dessas, referente a pesquisas com o nome científico da planta e 1 com o nome comum em território brasileiro. Para a base de dado internacional WIPO (World Intellectual Property Organization) contabilizou-se 2.680 depósitos com 196 patentes pertencentes à espécie Hibiscus sabdariffa e apenas 1 relativa a pesquisa pelo termo "vinagreira", que equivale à mesma patente depositada no INPI.

Tabela 2: Total de patentes registradas nas bases tecnológicas INPI e WIPO.

\begin{tabular}{c|cc}
\hline Palavras - chave & INPI & WIPO \\
\hline Hibiscus & 4 & 2.483 \\
Hibiscus sabdariffa & 3 & 196 \\
Vinagreira & 1 & 1 \\
Total & 8 & 2.680 \\
\hline
\end{tabular}

Conforme análise de patentes encontradas no INPI foi possível identificar 3 duplicatas de pedidos relacionados a espécie Hibiscus sabdariffa. Após o descarte desses pedidos, restaram somente 5 patentes, sendo 4 destas referentes ao gênero, 3 à espécie e 1 ao nome vulgar espécie vegetal. Para descrição de informações foram utilizados dados como o código do pedido, a data de depósito, o título e a CIP das patentes encontradas no INPI com a palavra-chave Hibiscus sabdariffa. No Quadro 2, observa-se que a primeira patente foi depositada no mês de fevereiro de 2016 e a segunda, em agosto de 2017. De acordo com a CIP, todas estão classificadas na seção A (necessidades humanas) com subclasses A23D (óleos ou gorduras comestíveis), A61K (preparações para finalidades médicas, odontológicas ou higiênicas) e A23L (alimentos, produtos alimentícios ou bebidas alcoólicas; seu preparo ou tratamento).

Quadro 2. Informações sobre patentes da espécie $H$. sabdariffa depositadas no INPI.

\begin{tabular}{|c|c|l|c|}
\hline Pedido & Depósito & \multicolumn{1}{|c|}{ Título } & CIP \\
\hline $\begin{array}{c}\text { BR10 2017 } \\
0175782\end{array}$ & $16 / 08 / 2017$ & $\begin{array}{l}\text { Composto de azeite de oliva extra virgem } \\
\text { aditivado com extrato concentrado de hibiscus } \\
\text { sabdariffa }\end{array}$ & A23D 9/007 \\
\hline $\begin{array}{c}\text { BR10 2017 } \\
0172260\end{array}$ & $10 / 08 / 2017$ & $\begin{array}{l}\text { Processo de obtenção de extrato líquido } \\
\text { concentrado do cálice de hibiscus sabdariffa } \\
\text { para preparo instantâneo de chás, bebidas e } \\
\text { outros usos alimentícios e produto assim }\end{array}$ & A61K 36/185 \\
\hline
\end{tabular}




\begin{tabular}{|c|l|l|c|}
\hline & & obtido & \\
\hline $\begin{array}{c}\text { BR10 2016 } \\
\text { 0024390 }\end{array}$ & $03 / 02 / 2016$ & $\begin{array}{l}\text { Bebida funcional de água de coco e chá de } \\
\text { hibisco (Hibiscus sabdariffa L.) }\end{array}$ & A23L 2/00 \\
\hline
\end{tabular}
Fonte: INPI, 2018.

Pode-se deduzir que a falta de investimentos do governo brasileiro no desenvolvimento de pesquisas tecnológicas influencia no baixo número de patentes depositadas em banco de dados nacionais, mesmo com o Brasil ocupando "o terceiro lugar no cenário mundial na produção de hortaliças e frutas" (HERRERA-ARELLANO et al., 2007). Quando comparado ao total de registros de pedidos em outros países, esse valor fica bem menos expressivo ainda.

A espécie Hibiscus sabdariffa é uma planta de adaptação de regiões tropicais e subtropicais tanto do hemisfério sul quanto do hemisfério norte (VIZZOTTO; PEREIRA, 2008). O Brasil é o país com a maior quantidade de florestas tropicais do mundo, apresentando a flora mais rica no cenário mundial. Entretanto, com relação ao cultivo e estudos de substâncias bioativas do hibisco no Brasil são poucas as recomendações ou investimentos de recursos para o desenvolvimento de patentes.

Com as informações coletadas na base de dado WIPO foi possível identificar 18 países detentores de patentes sobre a espécie Hibiscus sabdariffa a nível internacional, sendo que os principais foram escalados na Figura 6.

Figura 6: Países detentores de patentes da espécie H. sabdariffa.

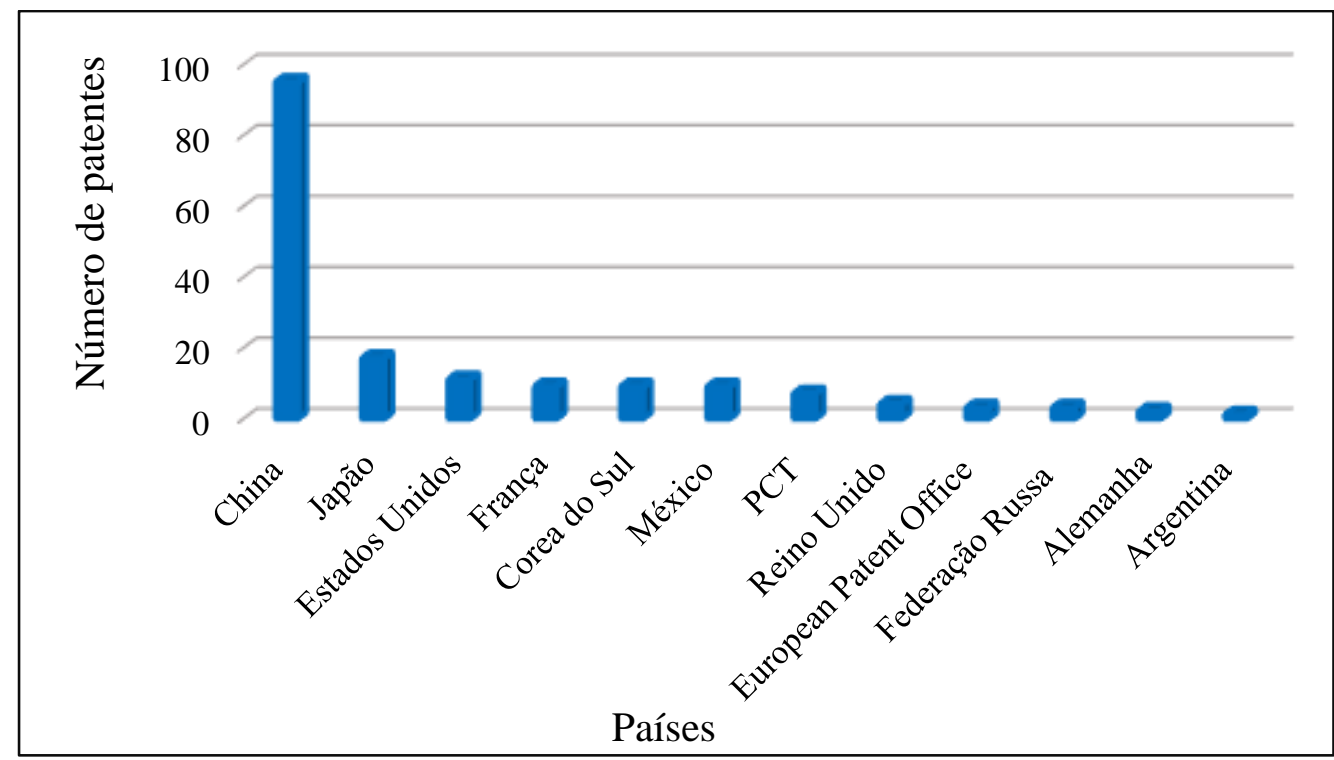

Fonte: Própria (2019)

$\mathrm{Na}$ Figura 6, observa-se que a China ocupa a primeira posição, com 95 patentes registradas e datadas de 1996 a 2017. Trata-se de uma quantidade expressiva, pois representa 
cerca de $53 \%$ do total registrado e demonstra a preocupação do país em proteger suas respectivas pesquisas. A segunda e terceira posição também são ocupadas por países de alto desenvolvimento tecnológico, como é o caso do Japão (17) e Estados Unidos (11). Logo em seguida, encontram-se França (9), Coreia do Sul (9), México (9), PCT (7), Reino Unido (4), EPO (3), Federação Russa (3), Alemanha (2) e Argentina (1).

A primeira patente sobre a espécie Hibiscus sabdariffa registrada na WIPO, foi depositada em outubro do ano de 1945 na Grã-Bretanha e de CIP C12P7/52, isto é, com classificação na seção C (Química; Metalúrgica) e subclasse C12P (processos de fermentação ou processos que utilizem enzimas para sintetizar uma composição/composto químico desejado ou para separar isômeros ópticos de uma mistura racêmica). Posteriormente, o segundo e terceiro depósitos, foram efetuados nos anos de 1980 na França e em 1996 na China. A evolução anual de pedidos de patentes até o ano de 2017 pode ser observada na Figura 7.

Figura 7: Número de patentes da espécie H. sabdariffa depositadas por ano.

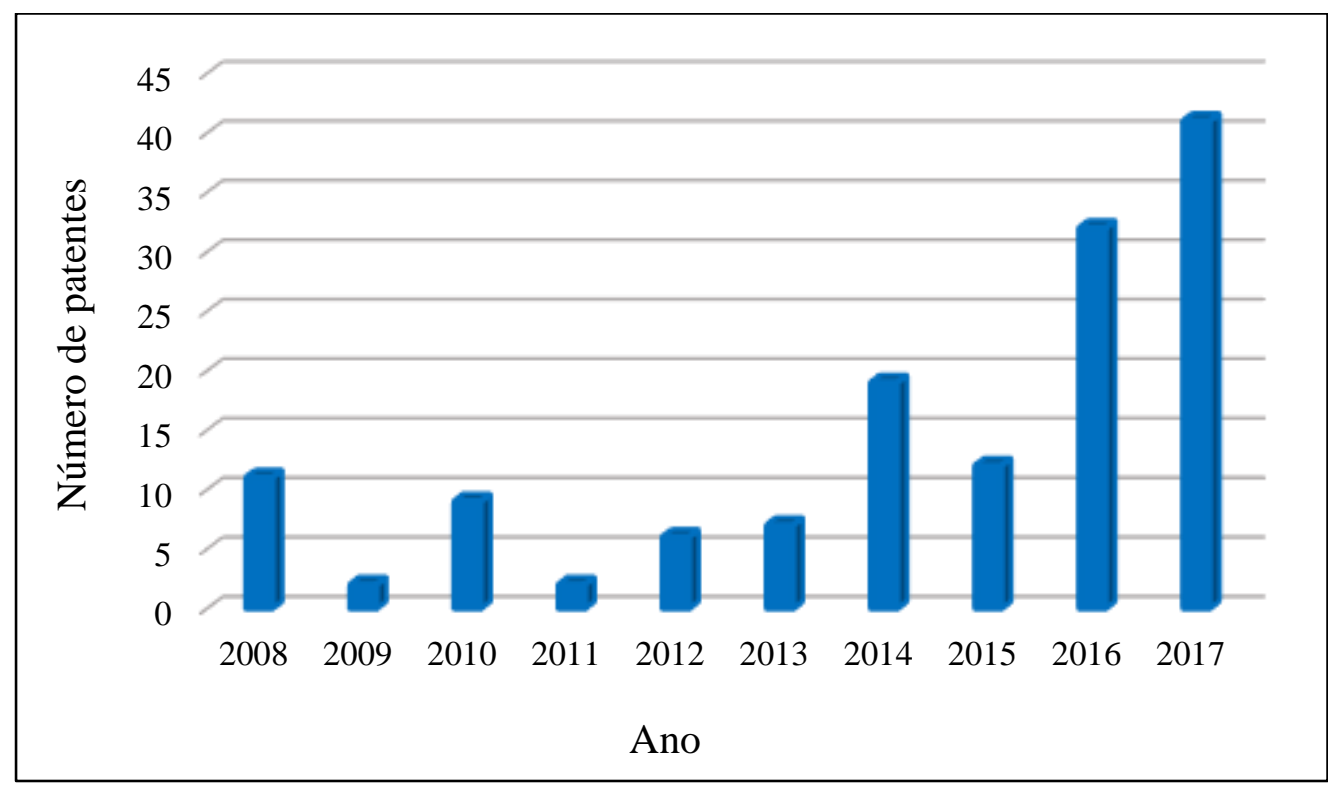

Fonte: Própria (2019)

Conforme observado na Figura 7, a quantidade de depósitos referentes aos anos de 2016 e 2017 se destacou em relação aos períodos anteriores, e assumem 41,5\% do total de registros até o último ano determinado para busca. Da quantia de 73 patentes reconhecidas nesse intervalo de tempo, 69 foram de pedidos feitos pela China, representando $91 \%$ dos documentos e ressaltando, novamente, a participação do governo chinês no investimento de pesquisas tecnológicas acerca da espécie Hibiscus sabdariffa. 
Segundo Gomes (2018), a patente atua como instrumento fundamental para as empresas garantirem proteção à criação e desenvolvimento de seus produtos. Além disso, permite também que o inventor tenha cobertura total sobre aspectos investigativos do invento. Nesse contexto, essa pesquisa identificou 10 principais empresas depositantes de patentes da espécie Hibiscus sabdariffa na base de dado WIPO.

Em primeiro lugar, com 4 documentos, encontra-se a empresa norte americana Mary Kay Intouch, com patentes distribuídas nas subclasses A61Q (uso específico de cosméticos ou preparações similares para higiene pessoal) e A61K (preparações para finalidades médicas, odontológicas ou higiênicas). Essa empresa foi criada em homenagem a fundadora Mary Kay Ash e tem por objetivo o desenvolvimento de "produtos nas categorias de maquiagem, cuidados com a pele e fragrâncias" (Mary Kay, 2018).

Figura 8: Principais empresas detentoras de patentes da espécie $H$. sabdariffa.

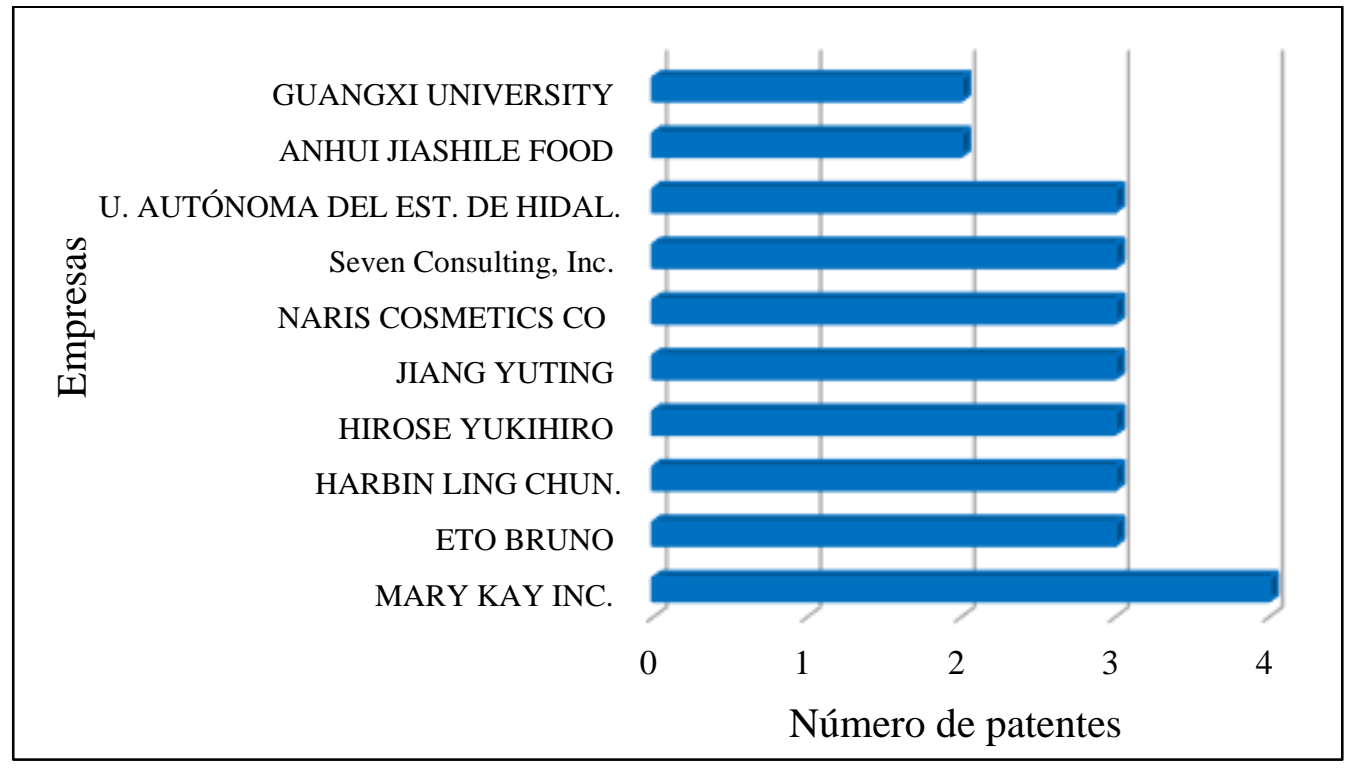

Fonte: Própria (2019)

Na sequência, com 3 documentos cada, destacam-se empresas/instituições chinesas (Harbin Ling Chun Wei Dao Food Development CO e Jiang Yuting), japonesas (Hirose Yukihiro e Naris Cosmetics CO), francesa (ETO Bruno), americana (Seven Consulting, Inc) e mexicana (Universidad Autónoma Del Estado De Hidalgo). Por fim, em terceira colocação com 2 documentos cada, estão a empresa Anhui Jiashile Food Processing CO e a Universidade Guangxi.

A CIP facilita a busca por patentes nas bases de dados tecnológicas, pois, as classifica de acordo com sua aplicação. Para análise de informações foi levado em consideração o fato de que uma determinada patente pode ter mais de uma classificação. De acordo com a Figura 
9 e baseando na CIP é possível identificar a organização das patentes relacionadas à espécie Hibiscus sabdariffa encontradas no banco de dados WIPO. O gráfico dessa figura fornece resultados quanto à seção, classe e subclasse. Assim, observa-se que a maioria das patentes foram enquadradas na seção A, sobre necessidades humanas, e outra parte na seção C, referente à aplicação na Química e Metalurgia.

Figura 9: Classificação das patentes quanto aos parâmetros da CIP.

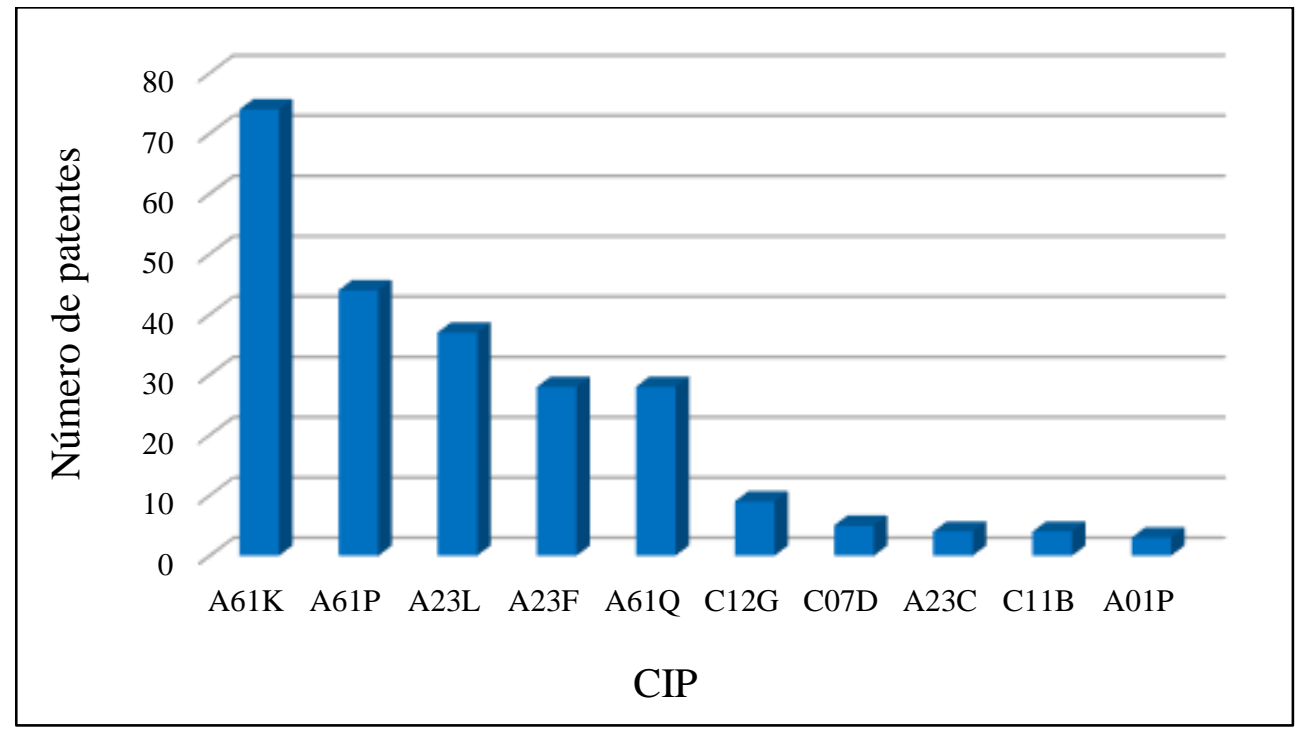

Fonte: Própria (2019)

As classes identificadas para a seção A foram: A61 (preparações para finalidades médicas, odontológicas ou higiênicas) como 146 patentes, isto é, cerca de 67\% do total encontrado; A23 (alimentos e seus produtos alimentícios; seu beneficiamento) com 69 patentes representando $31 \%$ em termos de porcentagem; A01 (agricultura; silvicultura; pecuária; caça; captura em armadilhas; pesca) com apenas 3 patentes.

Para a seção C foram registradas as seguintes classes: C12 (bioquímica; cerveja; álcool; vinho; vinagre; microbiologia; enzimologia; engenharia genética ou de mutação) com 9 patentes; C07 (química orgânica) com 5 patentes; C11 (óleos animais ou vegetais, gorduras, substâncias graxas ou ceras; ácidos graxos derivados dos mesmos; detergentes; velas) com 4 patentes.

A fim de descrever com mais detalhes informações sobre as subclasses presentes nas patentes, estas foram organizadas como segue no Quadro 4.

Quadro 3. Subclasses encontradas em patentes sobre a espécie H.sabdariffa

\begin{tabular}{|c|c|}
\hline $\begin{array}{c}\text { Subclasse } \\
\text { CIP }\end{array}$ & Título/Denominação \\
\hline A01P & Atividade de compostos químicos ou preparações biocidas, repelentes ou \\
\hline
\end{tabular}




\begin{tabular}{|c|l|}
\hline & atrativos de pestes ou reguladores do crescimento de plantas. \\
\hline \hline A23C & $\begin{array}{l}\text { Produtos de laticínio, p.Ex. leite, manteiga, queijo; substitutos do leite ou do } \\
\text { queijo; produção dos mesmos. }\end{array}$ \\
\hline \hline A23F & Café; chá; seus substitutos; manufatura, preparo, ou infusão dos mesmos. \\
\hline \hline A61K & $\begin{array}{l}\text { Café; chá; seus substitutos; manufatura, preparo, ou infusão dos mesmos; seu } \\
\text { preparo ou tratamento, p.Ex. cozimento, modificação das qualidades } \\
\text { nutritivas, tratamento físico; conservação de alimento ou produtos } \\
\text { alimentícios, em geral. }\end{array}$ \\
\hline \hline A61P & Preparações para finalidades médicas, odontológicas ou higiênicas. \\
\hline \hline A61Q & $\begin{array}{l}\text { Atividade terapêutica específica de compostos químicos ou preparações } \\
\text { medicinais. }\end{array}$ \\
\hline \hline C07D & Uso específico de cosméticos ou preparações similares para higiene pessoal. \\
\hline \hline C11B & $\begin{array}{l}\text { Produção, p. Ex. por compressão de matérias-primas ou por extração a partir } \\
\text { de substâncias de rejeitos, refinação ou preservação de óleos, substâncias } \\
\text { graxas, p.Ex. lanolina, óleos graxos ou ceras; óleos essenciais; perfumes. }\end{array}$ \\
\hline \hline C12G & Vinho; outras bebidas alcoólicas; sua preparação. \\
\hline
\end{tabular}

Mediante a quantidade expressiva de patentes encontradas e aplicadas em preparações de finalidades médicas, odontológicas ou higiênicas $(\mathrm{A} 61 \mathrm{~K})$, exploraram-se invenções recentes enquadradas nesse eixo. Dentre elas está um pedido depositado no ano de 2017 e de número WO2017105211, que utiliza um ácido extraído do caule do hibisco e seus derivados para aplicação na microbiologia, combatendo bactérias resistentes em meio vivo ou inerte.

O invento de identificação KR1020160114305, e igualmente classificado na CIP A61K, refere-se a um produto cosmético desenvolvido na Coréia do Sul e elaborado a partir do extrato de polifenóis presentes no Hibiscus sabdariffa que controla a temperatura da pele, evitando o envelhecimento precoce. Outro destaque na classificação $\mathrm{A} 61 \mathrm{~K}$ foi a patente depositada em 2012, pelo Instituto Mexicano de Seguro Social no México sob código de protocolo MX2012010586. Ao desenvolver esta pesquisa os inventores Alejandro Zamilpa Álvarez, Aime Tortoriello García e colaboradores criaram um produto à base do extrato aquoso de Hibiscus sabdariffa com metanol de acetonitrilo, que é aplicado no controle de algumas doenças musculares, tais como insuficiência renal, infarto e acidente vascular cerebral.

No campo de produtos com atividades terapêuticas específicas de compostos químicos ou preparações medicinais, isto é, de classificação A61P, ressalta-se a patente de protocolo CN105695102, arquivada no ano de 2016. Trata-se de mais uma pesquisa patenteada por uma instituição chinesa, a South China University Of Technology (Universidade de Tecnologia do 
Sul da China) que por meio de um método de separação a vapor, obteve o óleo do Hibiscus sabdariffa, o qual é utilizado na preparação de fármacos anti-inflamatórios. Em comparação as pesquisas de CIP A61P, identificou-se uma invenção importante no tratamento e inibição do câncer a partir do Hibiscus sabdariffa. O produto foi elaborado em maio de 2008 por Wang Chau-Jong nos Estados Unidos, com número de protocolo US20080113050. A patente refere-se à extração de antocianinas do hibisco para o trato do cancro em doentes com a infecção.

Para patentes de subclasse A23L, encontraram-se pesquisas tecnológicas importantes na aplicação do hibisco em preparo e conservação de bebidas nutritivas. É o caso da patente de protocolo CN201610898360.7, depositada pela China no ano de 2016, a qual utiliza Hibiscus sabdariffa, folhas de madressilva, hastes de hortelã e Fructus momordicae na preparação de um chá que pode restaurar a consciência, acalmar os nervos, remover o veneno do sangue, reduzir a pressão sanguínea e gordura e eliminar os radicais livres. Outro caso encontrado é o da pesquisa de protocolo PH22014000449, desenvolvida em 2014 nas Filipinas, a qual envolve o processo de produção do pó de rosélia (Hibiscus sabdariffa), uma fonte rica em nutrientes como a vitamina $\mathrm{C}$ e a antocianina, que pode ser aplicado como corante ou tempero de bebida alimentar.

Com relação à subclasse $\mathrm{C} 12 \mathrm{G}$, destacou-se outra patente depositada pela China no ano de 2017, sob número de protocolo CN2016112151726. A invenção trata-se de um vinho elaborado a partir da fruta do Hibiscus sabdariffa, o qual previne o acúmulo de gordura nas paredes internas das artérias, impedindo assim, acidentes vasculares, infartos e outros.

\section{Conclusões}

No panorama geral da prospecção científica a maioria das publicações referentes à espécie Hibiscus sabdariffa L. foram aplicadas na área da Farmacologia, Toxicologia e Farmácia e começaram a evoluir consideravelmente a partir do ano de 2011 até o ano de 2017, evidenciando assim, um avanço no número de pesquisas relacionadas à planta para os anos que se sucedem. A Nigéria, seguida da Índia e Taiwan foram considerados os países que mais publicaram sobre o hibisco nos últimos dez anos.

Com relação às supracitadas atividades farmacológicas, os polifenóis e antocianinas concentradas nos cálices, folhas e sementes do hibisco, apresentaram alto potencial medicinal na prevenção e no tratamento de doenças. No monitoramento da prospecção tecnológica, a 
China é a maior detentora de patentes. Assim, trata-se de um país que demonstra preocupação nas questões relacionadas à propriedade intelectual de pesquisas desenvolvidas em empresas/instituições do próprio país. Por outro lado, o Brasil apresentou resultados de baixas proporções na área de publicações científicas e no campo de produtos patenteados, refletindo a falta de investimentos do governo em pesquisas C\&T (Ciência e Tecnologia).

No referente ao código de classificação da CIP, as patentes encontradas se enquadraram nas seções A e C, com a maioria delas inclusas na subclasse A61K. Com isso, pode-se afirmar que, assim como é descrito em publicações científicas, a principal aplicação da espécie Hibiscus sabdariffa L. é em atividades com fins medicinais e farmacêuticos.

Considerando o fato de que quanto mais técnicas são descobertas, maior é a possibilidade da construção de novos saberes, esta pesquisa mostrou-se relevante e inovadora, pois através dos estudos de prospectivos, o conhecimento coletado sobre a espécie Hibiscus sabdariffa L. pode servir de base para pesquisadores que pretendem desenvolver trabalhos de inovação ou apenas obterem informações das características, composição química e propriedades farmacológicas da planta, além de proporcionar um panorama acerca dos avanços científicos dessa linha de pesquisa.

\section{Agradecimentos}

Ao Grupo de Pesquisa cadastrado no Conselho Nacional de Desenvolvimento Científico e Tecnológico (CNPq) em Alimentos, Química, Agronomia e Recursos Hídricos (AQARH).

\section{Referências}

AJAY, M.; CHAI, H.J.; MUSTAFA, A.M.; GILANI, A. H.; MUSTAFA, M. R. Mechanisms of the anti-hypertensive effect of Roselle L. calyces. Journal of Ethnopharmacology, v. 109, n. 3, p. 388-393, 2007.

ALARCÓN-ALONSO, J.; ZAMILPA, A.; ALARCÓN-AGUILAR, F.; HERRERA-RUIZ, M.; TORTORIELLO, J.; JIMENEZ-FERRER, E. Pharmacological characterization of the diuretic effect of Hibiscus sabdariffa Linn (Malvaceae) extract. Journal of Ethnopharmacology, v. 139, n. 1, 751-756, 2012.

ALI, B. H; CAHLIKOVÁ, L .; OPLETAL, L .; KARACA, T.; SULEIMANI, Y. A. L.; AL ZA'ABI, M. H.; BLUNDEN, G. Effect of aqueous extract of anthocyanins and calyces of Roselle (Malvaceae) in rats with adenine-induced chronic kidney disease. Journal of Pharmacy and Pharmacology, v. 69, n. 9, p. 1219-1229, 2017. 
ALI, B.H.; WABEL, N. A.; BLUNDEN, G. Phytochemical, Pharmacological and Toxicological Aspects of Hibiscus sabdariffa L.: A review. Phytotheraphy Research, v. 19, p. 369-375, 2005.

ALI, M. B; SALIH, W. M; MOHAMED, A. H; HOMEIDA, A. M. Investigation of the potential of Roselle calyces. Journal of Ethnopharmacoly, v. 31, n. 2, p. 249-257, 1991.

AL-MAMUN, A.; KHATUN, M. H.; NESSA, L.; ISLAM, M. D.; MUNIRA, S. In vitroevaluation of the antibacterial, cytotoxic and insecticidal activities of Hibiscus sabdariffafruits. Libyan Agriculture Research Center Journal Internation, v. 3, n. 1, 144$149,2011$.

AL-MAMUN; KHATUN, H.; NESA, L.; ISLAM, R.; MUNIRA, S. In vitro Evaluation of the Antibacterial, Cytotoxic and Insecticidal Activities of Hibiscus sabdariffa Fruits. Libyan Agriculture Research Center Journal Internation, v. 2, n. 3, p. 144-149, 2011.

CARDOSO, M. O. Vegetables unconventional Amazon. Brasilia: Embrapa-SPI. 1997.

CORADIN, L.; SIMINSKI, A.; REIS, A. Native species of flora of current or potential economic value: plans for the future - South Region Brasilia. 2011.

EZZAT, S. M.; SALAMA, M. M; SEIF EL-DIN, S. H; SALEH, S.; EL-LAKKANY, N. M.; HAMMAM, O. A.; SALEM, M. B.; BOTROS, S. S. Metabollic profile and hepatoprotective activity of the anthocyanin-rich extract of Hibiscus sabdariffa calyces. Pharmaceutical Biology, v. 54, n. 12, p. 3172-3181, 2016.

FULLERTON M. S.C.; VERGHESE, M.; WALKER, L. T.; SHACKELFORD, L. A.; CEBERT, E.; BOATENG, J.; OGUTU, S.; KHATIWADA, J.; GUYTON, M.; JONES, J.; CHAWAN, C. B. Determination of antioxidant contents in red sorrel and its anticarcinogenic potential in azoxymethane-induced colonic aberrant crypt foci. Research Journal of Phytochemistry, v. 2, n.1, p. 69-76, 2008.

GALVÃO, M. C. B. The bibliographic and scientific research. epidemiology fundamentals. 2018. 2nd ed. The 398, 1-377. Available at: <http://www2.eerp.usp.br/Nepien/DisponibilizarArquivos/Levantamento_bibliografico_Cristi aneGalv.pdf> Access: 15 February 2019.

GOMES, F. Understand the importance of patents for the creative industry. 2018. Available at <http://fgmarcas.com.br/entenda-a-importancia-das-patentes-para-a-industriacriativa-2/> Access: 10 March 2019.

GURROLA-DIÁZ, C. M.; GARCÍA-LÓPEZ, P. M.; SÁNCHEZ-ENRÍQUEZ, S.; TROYOSANROMÁN, R.; GONZÁLEZ-ANDRADE, I.; GÓMEZ-LEYVA, J. F. Effects of Hibiscus sabdariffa extract powder and preventive treatment (diet) on the lipid profiles of patients with metabolic syndrome (MeSy). Phytomedicine, v. 17, n. 1, p. 500-5005, 2010.

HERRERA-ARELLANO, A.; MIRANDA, S. J.; HERRERA, A. S.; JIMENEZ, F. J. E.; ZAMILPA, A.; ROMAN, R. R.; PONCE, M. H; TORTORIELLO, J. 2007. Clinical effects produced by the standardized herbal medicinal product of Roselle on Patients with 
hypertension. A randomized, double-blind, Lisinopril-controlled clinical trial. Planta Medica, v.73, p.6-12, 2007.

HIRUNPANICH, V.; UTAIPAT, A.; MORALES NP; BUNYAPRAPHATSARA, N.; SATO, H.; HERUNSALEE, A. Antioxidant effects of aqueous extracts from dried calyx of Hibiscus sabdariffa Linn. (Roselle) in vitro using rat low-density lipoprotein (LDL). Biological \& Pharmaceutical Bulletin, v. 28, n. 3, p. 481-484, 2005.

INPI. Patent Classification. 2018. Available at <http://www.inpi.gov.br/menuservicos/patente/classificacao-de-patentes> Access: 11 February 2019.

INUWA, I.; ALI, B. H; AL LAWATI, I .; BEEGAM, S .; ŽIADA, A.; BLUNDEN, G. Longterm ingestion of Roselle calyx extract Enhances myocardial capillarization spontaneously hypertensive rat in the. Experimental Biology and Medicine (Maywood), v. 237, n. 5, p. 563-569, 2012.

ISMAIL, A.; IKRAM, E. H. K; NAZRI, H. S. M. 2008 Roselle (Hibiscus sabdariffa L.) seeds nutritional composition protein quality and health benefits. Food, v. 2, n. 1, p. 1-16, 2008.

KAO, E. S.; YANG, M. Y.; HUNG, C. H.; HUANG, C. N.; WANG, C. J. Polyphenolic extract from Hibiscus sabdariffa reduces body fat by inhibiting hepatic lipogenesis and preadipocyte adipogenesis. Food Funct, v. 7, n. 1, p. 171-182. DOI: 10.1039/c5fo00714c.

KAPEPULA, $\quad$ P. M; KABAMBA-NGOMBE， N.; TSHISEKEDI-TSHIBANGU, P. TSUMBU, C.; FRANCK, T.; MOUITHYS-MICKALAD, A.; MUMBA, D.; TSHALAKATUMBAY, D.; SERTEYN, D.; TITS, M.; ANGENOT, L.; KALENDA, P. D. T.; FRÉDÉRICH, $\mathrm{M}^{1}$. Comparison of bioactivities and metabolic profiles of the leaves of three Congolese Hibiscus edible species. Natural Product Research, v. 31, n. 24, p. 2885-2892, 2017.

LEWINSOHON, T. M; PRADO, P. I. Brazilian Biodiversity: Current State of Knowledge Synthesis. 2018. Available at <http://www.mma.gov.br/port/sbf/chm/doc/estarte.doc> Access: 2 March 2018.

LIN, T .; LIN, M .; CHEN, C .; LIN, M .; CHOU, M .; WANG, C. Hibiscus sabdariffa extract angiograms serum cholesterol in men and women. Nutrition Research, v. 27, n. 3, p. 140$145,2007$.

LIU, J. Y; CHEN, C. C; WANG, W. H; H. S. U, J. D; YANG, M. Y; WANG, C. J. The protective effects of Hibiscus sabdariffa extract on CCl4-induced liver Brosis in rats. Food and Chemical Toxicology, v.44, p. 336-343, 2006.

LORENZI, H.; MATOS, F. J. A. Medicinal Plants in Brazil, Native and Exotic. Plantarum Institute of Plant Studies LTDA. São Paulo, 2002.

MOHAMMED, A.; FERNANDEZ, J.; PINEDA, M.; AGUILAR, M. Roselle (Hibiscus sabdariffa) seed oil is a rich source of (-) tocopherol. Journal of Food Science, vol. 72, p. 207-211, 2007. 
MOHD-ESA, N.; HERN, F. S; ISMAIL, A.; YEE, C. L. Indifferent parts Antioxidant activity of roselle (Hibiscus sabdariffa L.) extracts and exploitation potential of the seeds. Food Chemistry, v. 122, n. 4, 1055-1060, 2010.

MORTON, J. F. Roselle. In: Fruits of warm climates. Miami, USA: Florida Flair Books, p 2081-286, 1987.

OLALEYE, M. T. Cytotoxicity and antibacterial activity of Methanolic extract of Hibiscus sabdariffa. Journal of Medicinal Plants Research, v. 1, n. 1, p. 9-13, 2007.

OLALYE, M. T; ROCK, J. B. Commonly used medicinal plants tropical exhibit distinct in vitro antioxidant activities against hepatotoxins in rat liver. Experimental and Toxicologic Pathology, v. 58, n. 6, 433-438, 2007.

PAIM, M. P; MACIEL, M. J; WESCHENFELDER, S.; BERGMANN, G. P.; AVANCINI, C. A. M. Anti-Escherichia coli effect of Roselle L. In the meat model. Food Science and Technology, v. 37, v. 4, p. 647-650, 2017.

SARR, M.; NGOM, S.; KANE, M. O; WELE, A.; DIOP, D.; SARR, B. In vitro vasorelaxation mechanisms of bioactive compounds extracted from Roselle on rat thoracic aorta. Nutrition \& Metabolism, v. 6, n. 45, p. 1-12, 2009.

VIZZOTTO, M.; PEREIRA, M. C. Hibisco: do uso ornamental ao medicinal. Infobibos Informações Tecnológicas. 2008. 